\title{
STUDY OF THE VARIABLES THAT INFLUENCE IN SITU TRANSESTERIFICATION REACTION OF DESMODESMUS SP.
}

\section{Vinícius F. Vieira*, Luisa F. Rios Pinto, Gabriela F. Ferreira and Leonardo V. Fregolente.}

\begin{abstract}
In recent years, the cultivation of microalgae for biofuels production has been extensively studied. However, the conventional production of microalgae oil involves high investments and cost of operation. One promising alternative is the in situ transesterification because it excludes the oil extraction step. In this work, it was proposed the study of biodiesel production from microalga Desmodesmus sp. The effects of temperature, reaction time, alcohol volume, and percentage of catalyst on the yield of fatty acid methyl esters were evaluated. The results showed that the reaction temperature parameter is the most significant in obtaining esters.
\end{abstract}

\section{Key words:}

Microalgae, in situ transesterification, biodiesel.

\section{Introduction}

In situ or direct transesterification began to be studied in 1985 and consists of using the reagent alcohol itself as a solvent, without the prior extraction of the oil. The temperature, the percentage of the catalyst, the reaction time, and the volume of alcohol that lead to the optimal reaction conditions depend heavily on the microalgae species and the corresponding lipid profile.

In this way, it intends to investigate, through statistical analysis, the conditions for better performance of in situ transesterification reaction of the oil present in the biomass of Desmodesmus sp.

\section{Results and Discussion}

For the statistical analysis of the factors effect on the yield, a fractional factorial design of experiments with two levels was set up, with triplicate in the central point. Chart 1 presents the variables values of each run with yield in $\mathrm{mg}$ ester/gbiomass.

Chart 1. Variables values and yield.

\begin{tabular}{cccccc}
\hline \hline Runs & $\begin{array}{c}\text { Catalyst } \\
(\mu \mathrm{L})\end{array}$ & $\begin{array}{c}\text { Temperature } \\
\left({ }^{\circ} \mathrm{C}\right)\end{array}$ & $\begin{array}{c}\text { Alcohol } \\
(\mathrm{mL})\end{array}$ & $\begin{array}{c}\text { Time } \\
(\mathrm{h})\end{array}$ & Yield \\
\hline \hline 1 & 54 & 70 & 3 & 0.50 & 58.31 \\
2 & 218 & 70 & 3 & 7.00 & 49.79 \\
3 & 54 & 140 & 3 & 7.00 & 77.62 \\
4 & 218 & 140 & 3 & 0.50 & 82.80 \\
5 & 54 & 70 & 7 & 7.00 & 29.32 \\
6 & 218 & 70 & 7 & 0.50 & 71.69 \\
7 & 54 & 140 & 7 & 0.50 & 85.78 \\
8 & 218 & 140 & 7 & 7.00 & 58.82 \\
$9(\mathrm{C})$ & 136 & 105 & 5 & 3.75 & 34.24 \\
$10(\mathrm{C})$ & 136 & 105 & 5 & 3.75 & 43.51 \\
$11(\mathrm{C})$ & 136 & 105 & 5 & 3.75 & 46.39 \\
\hline \hline
\end{tabular}

From the analysis in Statistica ${ }^{\circledR}$ software, disregarding the interactions between variables and considering only pure error, once the replicates were only made for the central point, in the table of effects estimates, as well as the Pareto graph, shown in Image 1, it was obtain the most significant factors, in a confidence interval of $95 \%$ ( $p$-value $=0.05$ ), temperature and time.

Image 2 shows the marginal means of these factors, where it can see the positive effect of temperature and the negative effect of time on yield. The approximately parallel lines in the graph suggest that there is no significant interaction between time and temperature.
Image 1. Pareto Chart of Effects.

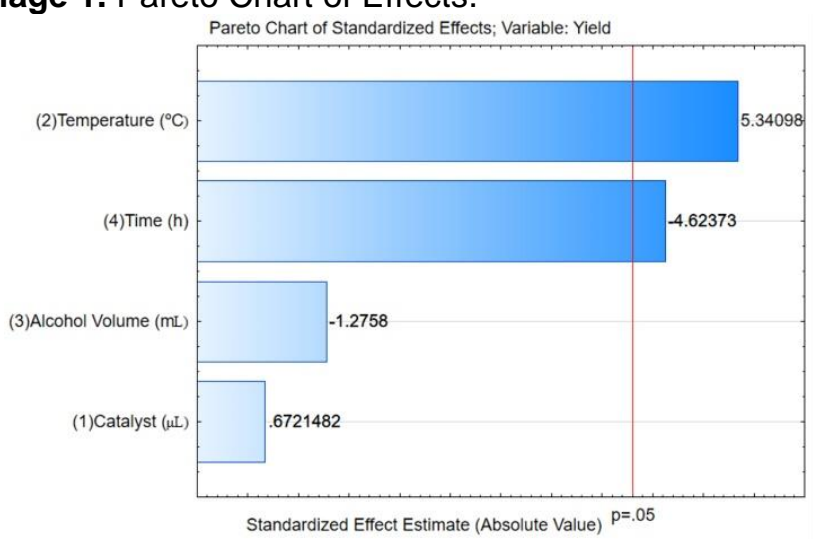

Image 2. Marginal means and Conf. Limits (95\%)

Plot of Marginal Means and Conf. Limits (95\%) NOTE: Std.Errs. for means computed from MS Error $=40.3083$

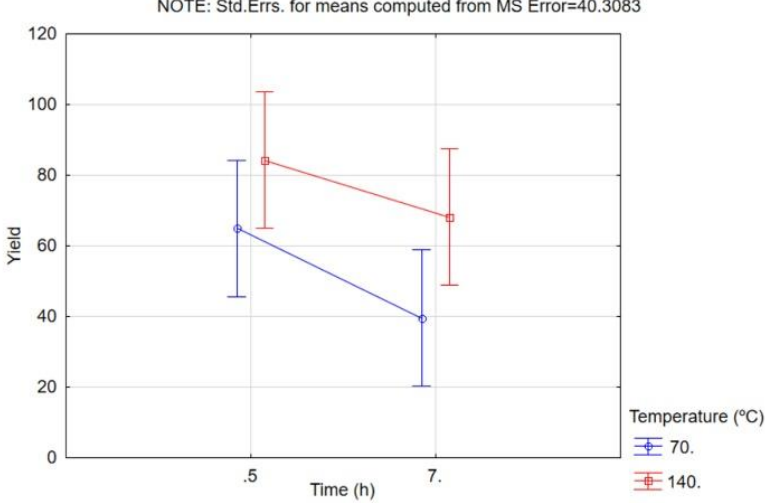

\section{Conclusions}

The temperature of the reaction was the most significant variable in biodiesel production and the reaction time had a significant negative effect, as the highest yields were obtained under the conditions of $140{ }^{\circ} \mathrm{C}$ and the minimum level of time $(0.5 \mathrm{~h})$. The obtained model approached the results obtained experimentally, within the standard error. However, the analysis also shows that a non-linear model would be more significant; therefore, further experiments and analyzes are necessary.

\section{Acknowledgement}

The authors are grateful for the funding of CNPq together with the PIBIC/UNICAMP program for support to research and The São Paulo Research Foundation, Processes No 2014/10064-9 and 2015/20630-4. 